\title{
Superlubricity under ultrahigh contact pressure enabled by partially oxidized black phosphorus nanosheets
}

\author{
Xiaoyong Ren ${ }^{1,2}$, Xiao Yang $\mathbb{D}^{1,2}$, Guoxin Xie $\mathbb{D}^{1 凶}{ }^{凶}$, Feng He${ }^{1}$, Rong Wang ${ }^{1}$, Chenhui Zhang ${ }^{1}$, Dan Guo ${ }^{1}$ and Jianbin Luo ${ }^{1 凶}$
}

Superlubricity has recently raised an increasing interest owing to its great potential in energy saving and environmental benefits. Yet how to obtain stable superlubricity under an ultrahigh contact pressure ( $>1 \mathrm{GPa}$ ) still remains a challenge. Here, we demonstrate that robust liquid superlubricity can be realized even under a contact pressure of 1193 MPa by lubrication with partially oxidized black phosphorus (oBP) nanosheets. The analysis indicates that the oBP nanosheets that absorb large amounts of water molecules are retained at the friction interface and transform the friction pairs interface to that between the oBP nanosheets. Molecular dynamics simulation demonstrates that water molecules could be retained at the friction interface even under the ultrahigh contact pressure owing to the abundant $\mathrm{P}=\mathrm{O}$ and $\mathrm{P}-\mathrm{OH}$ bonds formed on the oBP nanosheet surfaces, contributing to the achievement of stable superlubricity under the ultrahigh contact pressure. This work has the potential of introducing the liquid superlubricity concept in diverse industrial applications involving high-contact-pressure operating conditions.

npj 2D Materials and Applications (2021)5:44; https://doi.org/10.1038/s41699-021-00225-0

\section{INTRODUCTION}

Superlubricity, a state in which friction completely or nearly vanishes between the contacting surfaces in relative motion, is highly desirable for energy savings, environmental protection, and enhancement of the lifetime of mechanical components ${ }^{1-3}$. To date, the superlubricity state has been realized in a limited number of dry contacting solid surfaces (solid superlubricity) or with the use of appropriate liquids at the liquid-lubricated contacts (liquid superlubricity) ${ }^{2}$. Solid superlubricity has been demonstrated for a couple of materials, e.g., molybdenum disulfide $\left(\mathrm{MoS}_{2}\right)^{4,5}$, highly oriented pyrolytic graphite ${ }^{6}$, multiwalled carbon nanotubes ${ }^{7}$, graphene ${ }^{8-11}$, hexagonal boron nitride ${ }^{12}$, and diamond-like carbon films ${ }^{13}$. Solid superlubricity is normally pertinent to the incommensurate structural phase of the two surfaces $^{2}$. It is mainly achieved at nano- or microscale owing to the inevitable structural deformation or defects in macroscale materials. Meanwhile, specific lubrication conditions were normally the requisites for solid superlubricity, such as dry nitrogen atmosphere or ultrahigh vacuum. Most of the studies about solid superlubricity have focused on realizing it at larger scales and under ambient conditions, and considerable progresses have been made so $\mathrm{far}^{2,14,15}$. Compared with solid superlubricity, liquid superlubricity could be achieved at different scales and under ambient conditions. In contrast, the main challenge in liquid superlubricity is as to how to realize it under high-contact pressures since the contact pressure is relatively low in most of the liquid superlubricity systems ${ }^{2}$. It is reported that the solid superlubricity was attained at microscale under contact pressure up to $1 \mathrm{GPa}^{16}$ and was attained at nanoscale under ultrahigh contact pressure of up to $2.52 \mathrm{GPa}$, by the formation of transferred graphene nanoflakes on a silicon tip ${ }^{17}$. However, it has been reported that the contact pressures are up to $\sim 7.5, \sim 10$, and $\sim 300 \mathrm{MPa}$ in the liquid lubrication systems of polymer brushes ${ }^{18}$, water-lubricated self-mated ceramics ${ }^{19}$, and polyhydroxy alcohol/ acid mixtures ${ }^{20,21}$, respectively. Although the contact pressures in the above systems could satisfy some of the applications (such as human-made joints), the attempts to obtain stable superlubricity under a higher contact pressures in consideration of the widespread high-contact-pressure operating conditions in industrial applications are required ${ }^{18,22}$. To this end, many researches focused on accomplishing liquid superlubricity at high-contact pressures, but the contact pressures used in these studies have rarely been over $300 \mathrm{MPa}^{2,18-22}$.

Black phosphorus (BP) has attracted considerable attention because of its anisotropic lamellar structure, tunable directbandgap ( $0.3 \mathrm{eV}$ for bulk and $1.5-2 \mathrm{eV}$ for monolayer) and high carrier mobility $\left(\sim 1000 \mathrm{~cm}^{2} \mathrm{~V}^{-1} \mathrm{~s}^{-1}\right)^{23}$. The oxidation and lightinduced degradation of BP is an undesirable but familiar process, resulting in the rapid loss of semiconducting properties, which limits the potential applications of BP in electronics and optoelectronics ${ }^{24}$. However, it is demonstrated that by coupling nanosheets of oxidized $\mathrm{BP}$ and reduced graphene oxide (GO), the surface redox pseudocapacitance exceeds the kinetic and stability limitations of previously explored $\mathrm{BP}^{23}$. This study opens an avenue of research on the use of oxidized BP, although most of the studies have focused on how to passivate BP to avoid the oxidation. The effects of degradation usually result in the formation of liquid droplets on the surface of the $\mathrm{BP}$ flake ${ }^{25}$. To some extent, this phenomenon reflects the strong water adsorption capacity of oxidized BP, which inspires us to investigate this aspect of liquid superlubricity, because water is one of the most suitable lubricants in liquid superlubricity owing to its low cost and viscosity ${ }^{2,26-28}$. Indeed, early experiments by using atomic force microscopy (AFM) indicated, for instance, the friction force reduced by about $50 \%$ at the degraded area of the BP nanosheets and a super-slippery interface was observed between the degraded $\mathrm{BP}$ and the $\mathrm{SiO}_{2}$ substrate ${ }^{29,30}$. An oxidized BP-based liquid superlubricity system seems, therefore, feasible and expected to provide liquid superlubricity under a higher contact pressure.

In the light of these considerations, the present study focuses on using actively oxidized black phosphorous (oBP) nanosheets as the lubricant additive. The experiments reveal that a robust liquid superlubricity state can be realized even under a contact pressure

\footnotetext{
${ }^{1}$ State Key Laboratory of Tribology, Department of Mechanical Engineering, Tsinghua University, Beijing, China. ${ }^{2}$ These authors contributed equally: Xiaoyong Ren, Xiao Yang.

凶email: xgx2014@tsinghua.edu.cn; luojb@tsinghua.edu.cn
} 
of $1193 \mathrm{MPa}$. Transmission electron microscopy (TEM), electronenergy loss spectroscopy (EELS), X-ray photoelectron spectroscopy (XPS), and Raman spectroscopy were used to explore the physical and chemical properties of the wear surface to gain insights into the superlubricity mechanism under the extreme contact pressure with the help of partially OBP nanosheets. Molecular dynamics (MD) simulations were also performed to further elucidate the mechanisms at a fundamental level. A deeper understanding of the superlubricity mechanism is an essential milestone toward the establishment of liquid superlubricity systems under ultrahigh contact pressures.

\section{RESULTS AND DISCUSSION}

Preparation and characterization of partially oBP nanosheets

Figure 1 shows the schematic illustration of the synthesis process of the suspension with partially OBP nanosheets. The BP powder used was prepared through the high-energy ball-milling technique by using red phosphorus (RP) as the starting material. The typical morphologies, X-ray diffraction (XRD) patterns and Raman spectra of the obtained BP and raw RP powders are shown in Supplementary Fig. 1. Dilute hydrogen peroxide (HP) solution was used as the oxidizing agent. Firstly, a controlled amount of HP solution ( $30 \mathrm{wt} \%$ ) was added into the deionized water to make the dilute solution. Then, the bulk BP powder was dispersed into the dilute solution and stirred for $30 \mathrm{~min}$. Subsequently, the obtained solution was ultrasonically treated for another $2 \mathrm{~h}$ in the ice-bath. Finally, the unexfoliated BP was removed by centrifugation at $2000 \mathrm{rpm}$ for $20 \mathrm{~min}$ to yield a light brown supernatant. Since $\mathrm{H}_{2} \mathrm{O}_{2}$ is a kind of strong oxidizing agent and normally used as decolorizer, the ratio of $\mathrm{H}_{2} \mathrm{O}_{2}$ and $\mathrm{BP}$ was controlled. Supplementary Fig. 2 shows the color changes of the $\mathrm{BP}$ aqueous suspensions with time after adding different amounts of $\mathrm{H}_{2} \mathrm{O}_{2}$. The results indicated that the higher the ratio between $\mathrm{H}_{2} \mathrm{O}_{2}(\mu \mathrm{l})$ and $\mathrm{BP}(\mathrm{mg})$ was, the faster the degradation of oBP. The oBP suspension with the ratio of 1.5 could be kept stable for several days. Thus, the ratio of $\mathrm{H}_{2} \mathrm{O}_{2}(\mu \mathrm{l})$ and $\mathrm{BP}(\mathrm{mg})$ was set as 1.5 in this work.

We confirmed that the oBP nanosheets could be formed with the oxidation of a dilute HP solution and subsequent ultrasonic exfoliation. The size of the nanosheets is up to $\sim 12 \mu \mathrm{m}$ in length, $\sim 6 \mu \mathrm{m}$ in width, and $\sim 20 \mathrm{~nm}$ in thickness, as shown in Fig. 2a. Considering the thickness of single-layer BP, $\sim 0.53 \mathrm{~nm}$, together with the roughness of substrate and the absorbed water molecules on the surface, we deduce that the oBP nanosheet is $<37$ layers. Supplementary Fig. 3 shows the optical and scanning electron microscopy (SEM) images of the oBP nanosheets. The optical image shows that the oBP nanosheets adsorb water molecules easily, resulting in some droplets on them. The SEM images indicate that the oBP nanosheets surfaces are not smooth and some bubbles are present on them (Supplementary Fig. 3b and Fig. 2b). The morphologies are similar to that of the BP flakes after exposure for days in ambient conditions, resulting from the degradation of BP flakes by oxygen and water ${ }^{29,31,32}$. The corresponding energy dispersive spectroscopy (EDS) mapping spectra show the presence of $\mathrm{P}$ and $\mathrm{O}$ elements on the nanosheet surface. Meanwhile, the feature peaks of the BP crystal are detected in the XRD patterns of OBP nanosheets, although the intensities of the feature peaks are relatively weak, compared with that of the initial BP powder or the powder just treated with water (Supplementary Fig. 4a). The detection of the characteristic peaks of BP confirms the preservation of the BP crystal structure to some extent, indicating that BP is partially oxidized. The thermal gravimetric analysis (TGA) results (Supplementary Fig. 4b) also confirm the presence of the restrained water in the oBP nanosheets. Compared with the BP powder, the oBP nanosheets show $15 \%$ weight loss from room temperature to $400^{\circ} \mathrm{C}$, while the corresponding weight change of the BP powder is very little. The retained water on the oBP surface is supposed to be able to stay at the friction interface even under a high-contact pressure, which is helpful to realize superlubricity under the high-contact pressure.

The morphologies obtained with the TEM images indicate that the oBP nanosheets are porous (Fig. 2c). The micro-pores are homogeneously and widely distributed not only on the oBP nanosheet surface, but also in the interlamination. The EDS mapping indicates the homogeneous distribution of the $\mathrm{P}$ and $\mathrm{O}$ elements on the oBP nanosheets and the distribution position corresponding to the porous morphology (Supplementary Fig. $5 a-c)$. However, when the line-scanning analysis of the pore areas was carried out, we found that these porous structures were very sensitive to electron irradiation (Supplementary Fig. $5 \mathrm{~d}-\mathrm{f}$ ). Therefore, the in situ morphological changes of oBP nanosheets with the long-time electron beam irradiation in vacuum are observed (Supplementary Fig. 6). It is shown that the pores are small, homogeneous and there is wide distribution in the oBP nanosheets on the first look. However, with the increase in the electron beam irradiation time, the pores gradually diffuse, coalesce, and get removed with the shrinkage of the oBP nanosheet size. After $5 \mathrm{~min}$ irradiation, most of the porous structures disappear and the size of the oBP nanosheet becomes stable. The oxidized BP nanosheets absorb and retain water molecules on the surfaces ${ }^{32,33}$, and the porous structure may be caused by the evaporation of the retrained water in the highvacuum condition. The porous structures in the interlamination of the oBP nanosheets indicate that the oxidation not only occurred at the topmost surface but also in the layers beneath, which is different from the oxidation process of BP flakes in ambient

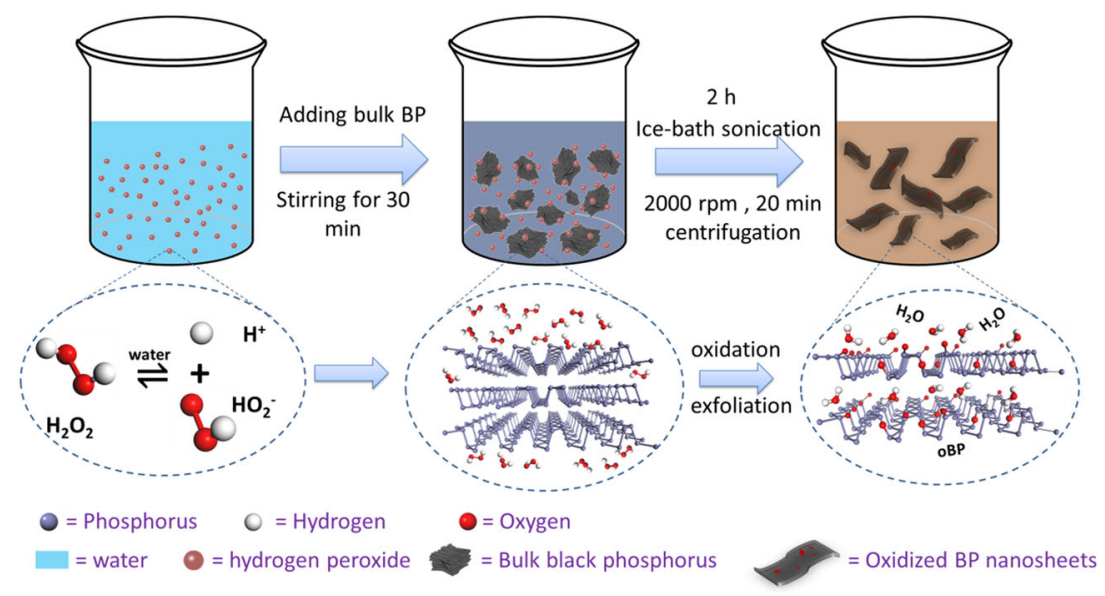

Fig. 1 Schematic illustration. Schematic illustration of the synthesis process of the suspension with partially oBP nanosheets. 
a

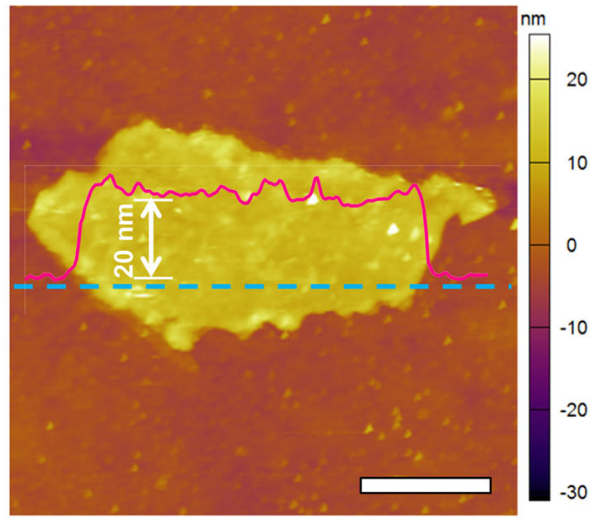

c

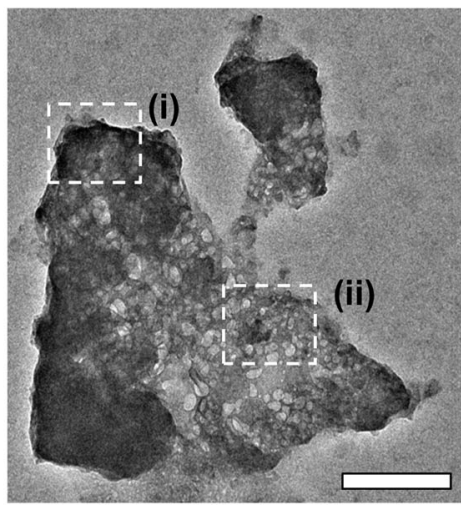

e

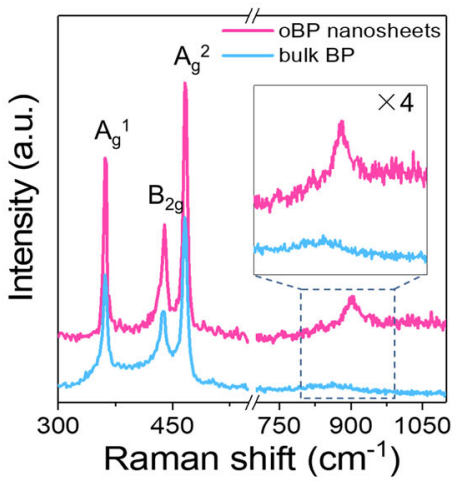

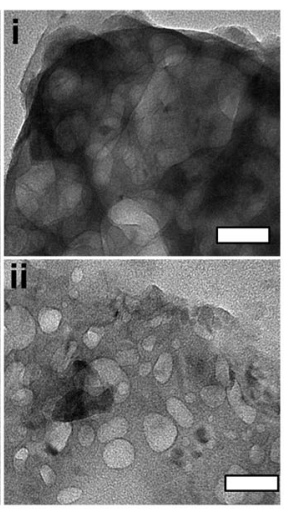

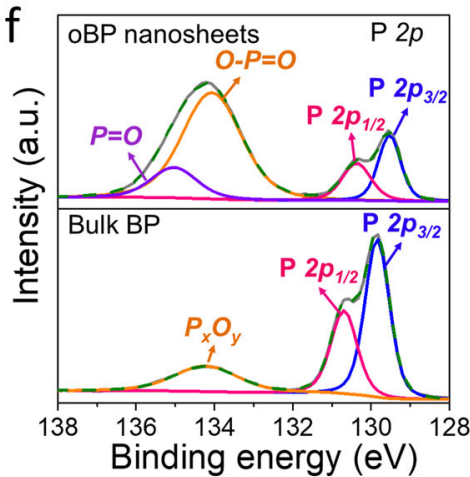

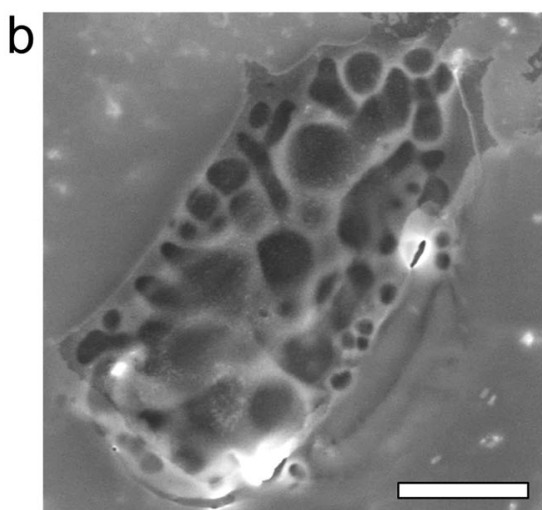
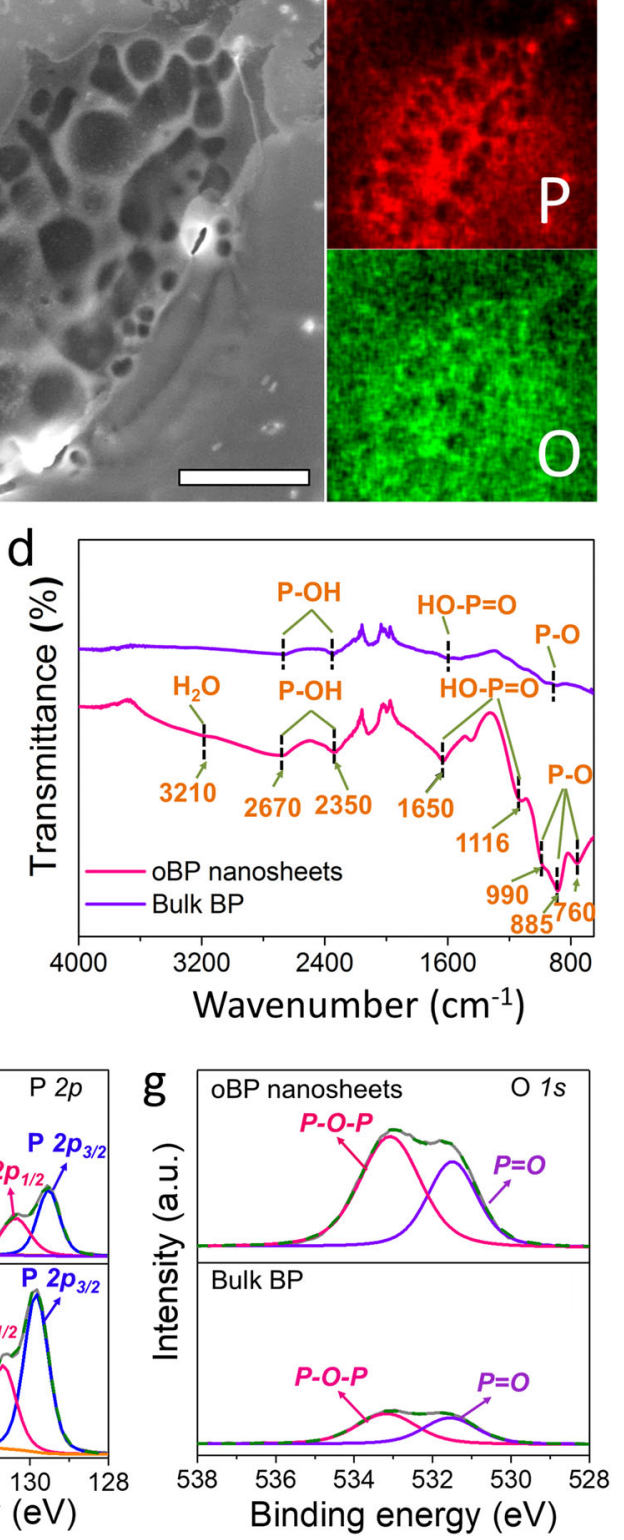

Fig. 2 Structural characterizations of the oBP nanosheets oxidized by dilute HP. a AFM image and the height profile of the oBP nanosheet. Scale bar: $5 \mu \mathrm{m}$. b SEM image of the oBP nanosheet and the corresponding EDS mapping spectra. Scale bar: $5 \mu \mathrm{m}$. c TEM image of the oBP nanosheet. Scale bar: $200 \mathrm{~nm}$. (i and ii) The amplification images of the corresponding parts in c. Scale bar: $50 \mathrm{~nm}$. d FTIR spectra of the oBP nanosheets and the bulk BP powder $\left(600-4000 \mathrm{~cm}^{-1}\right)$. e Raman spectra of the oBP nanosheets and the bulk BP powder $\left(300-1100 \mathrm{~cm}^{-1}\right)$. f XPS spectra of P $2 p$ and $(\mathbf{g}) \mathrm{O} 1 \mathrm{~s}$ of the oBP nanosheets and the bulk BP powder.

conditions (mainly occurred at the topmost layer) ${ }^{32,33}$. The active oxidation of the BP nanosheets by HP is faster and more homogeneous than that in ambient conditions. The observed porous structures on the surface and in the interlamination of the OBP nanosheets have been attributed to the pitting oxidation mechanism, which is helpful to retain abundant water molecules during the lubrication process $\mathrm{s}^{29,32}$.

Fourier-transform infrared (FTIR) spectra were used to confirm the partially oxidized state of the oBP nanosheets (Fig. 2d). For the bulk BP powder, the weak absorption bands at 890, 1650, and $2350 \mathrm{~cm}^{-1}$, which were assigned to the $\mathrm{P}-\mathrm{O}, \mathrm{P}=\mathrm{O}$, and $\mathrm{P}-\mathrm{OH}$ bonding, were detected owing to the slight oxidization of $\mathrm{BP}$ powder during the sample preparation and characterization. Compared with the bulk BP powder, the oBP nanosheets indicate the obvious absorption bands centered at about 760, 885, and $990 \mathrm{~cm}^{-1}$, which were assigned to the $\mathrm{P}-\mathrm{O}$ bonds, signifying the formation of $\mathrm{P}_{x} \mathrm{O}_{y}$ in the nanosheets ${ }^{29}$. The absorption bands at about 1116 and $1650 \mathrm{~cm}^{-1}$ were assigned to the symmetric stretching modes of the $\mathrm{P}=\mathrm{O}$ bonds ${ }^{34}$. The $\mathrm{P}-\mathrm{OH}$ bonds were detected at about 2350 and $2670 \mathrm{~cm}^{-1}$, indicating the hydroxyl group existed in the oBP nanosheets ${ }^{34}$. At the same time, the $\mathrm{H}_{2} \mathrm{O}$ adsorption band at $3210 \mathrm{~cm}^{-1}$ was also detected ${ }^{35}$.

To further identify the variation of the chemical groups after oxidation, Raman spectroscopic measurements were conducted on the bulk $\mathrm{BP}$ and the oBP nanosheets with a wave number range from 300 to $1100 \mathrm{~cm}^{-1}$. For the oBP nanosheets, three characteristic peaks, located at $361.3,439.4$, and $465.9 \mathrm{~cm}^{-1}$ were detected, which were assigned to $A_{g}{ }^{1}$ (out-of-plane), $B_{2 g}$ (in-plane), and $A_{g}{ }^{2}$ (in-plane) vibrational modes of $B P$, respectively (Fig. $2 e)^{36}$. It is well known that BP is anisotropic material where its vibrational modes are sensitive to the number of layers especially at the nanoscale ${ }^{23}$. The $A_{g}{ }^{2}$ peak position of the oBP nanosheets shifted $1.6 \mathrm{~cm}^{-1}$ to the higher wavenumber compared with the bulk BP powder, indicating the successfully exfoliation of oBP nanosheets. 
Moreover, the peak at about $902 \mathrm{~cm}^{-1}$ became obvious, which was mainly assigned to the Raman active modes of phosphorus oxides $^{30}$. Therefore, the partially oxidized state of the BP nanosheets is demonstrated by the Raman spectra.

The oxidization of the BP nanosheets could also be confirmed by using XPS measurements. Figure $2 f, g$ shows the obtained $P 2 p$ and $\mathrm{O}$ 1s core-level spectra of the bulk BP powder and the oBP nanosheets. The bulk BP powder indicated a P $2 p$ doublet of $2 \mathrm{p}_{1 / 2}$ and $2 p_{3 / 2}$ at 129.9 and $130.8 \mathrm{eV}$ (spin-orbit splitting of $\sim 0.9 \mathrm{eV}$ ) and a broad peak at $134.7 \mathrm{eV}$. The former $\mathrm{P} 2 \mathrm{p}$ peaks corresponded to the unreacted $\mathrm{BP}$, and the latter broad peak to the residual phosphorus oxides (mainly $\mathrm{O}-\mathrm{P}=\mathrm{O}$ bond) ${ }^{23,29}$. In spite of the very short duration of ambient exposure during the XPS sample preparation, the bulk BP powders undergo ambient oxidation to some extent ${ }^{23,29}$. The weak peaks at 531.2 and $533.5 \mathrm{eV}$ in the $\mathrm{O} 1 \mathrm{~s}$ spectrum of the bulk BP powder also indicate the presence of BP oxide and products, being consistent with the $P 2 p$ spectrum. For the oBP nanosheets, the intensity of the P-P bonds for BP reduced, while that of the peak for phosphorus oxides increased. The peaks appear at 134.1 and $135 \mathrm{eV}$, which can be assigned to the dangling $(\mathrm{P}=\mathrm{O})$ and $\mathrm{O}-\mathrm{P}=\mathrm{O}$ phosphorus-oxygen bonds, owing to the oxidation of $\mathrm{BP}^{23,29}$. The $\mathrm{O} 1 \mathrm{~s}$ spectrum of the oBP nanosheets in Fig. $2 \mathrm{~g}$ shows the substantial growth of the two main peaks ( 531.5 for $\mathrm{P}=\mathrm{O}$ dangling oxygen and $533.2 \mathrm{eV}$ for bridging oxygen) ${ }^{23,29}$. The relative atomic ratio of total $\mathrm{O}$ to oxidized $\mathrm{P}(134-135 \mathrm{eV})$ for the oBP nanosheets is 1.67 , which was between the stoichiometric ratio of 1.5 (for $\mathrm{P}_{2} \mathrm{O}_{3}$ ) and 2.5 (for $\mathrm{P}_{2} \mathrm{O}_{5}$ ). The results confirm the partially oxidized state of the oBP nanosheets.

The dangling oxygen $(\mathrm{P}=\mathrm{O})$ changes the surface state and serves as the linkage point for the hydrogen bonds with water molecules, resulting in the transition of the $\mathrm{BP}$ surface from hydrophobic to hydrophilic ${ }^{23,30,37}$. According to the first-principles calculations, the binding energy between the water molecule and the $\mathrm{P}=\mathrm{O}$ group on the oBP nanosheet surface (about $-0.1914 \mathrm{eV}$ ) is larger than that between the water molecule and the pristine BP surface (about $-0.1870 \mathrm{eV})^{30}$. At the same time, the $\mathrm{H}_{(\mathrm{H} 2 \mathrm{O})}-\mathrm{O}_{(\mathrm{P}=\mathrm{O})}$ bonding length $(2.462 \AA)$ is lower than the typical hydrogen-bond length $(\sim 2.5 \AA)$ between water molecules, indicating a stable restrained water layer bound by the hydrogen bonds that may form on the oBP nanosheets surface ${ }^{30}$.

\section{Friction studies}

The macroscale liquid superlubricity behaviors under ultrahigh contact pressures with the OBP nanosheets suspensions are demonstrated in Fig. 3a, b. The corresponding coefficient of friction (COF) dropped from $\sim 0.1$ to $0.004 \sim 0.007$ after the runningin period, and then fluctuated slightly, maintaining the state of superlubricity $(\mathrm{COF}<0.01)$. In comparison, the COFs for pure water, a dilute HP solution, and a BP particle suspension (Supplementary Fig. 7a) increased from 0.02 to 0.04 to a high level of 0.1-0.2 after the running-in period. The sudden increase (for water, the HP solution, and the BP particle suspension) or decrease (for the oBP nanosheets suspension) of the COFs could be attributed to the evaporation of water from the lubricants to some extent. To prove this hypothesis, the variation of the COFs lubricated with different volumes of lubricants at $25^{\circ} \mathrm{C}$ or at different test temperatures with a fixed volume $(20 \mu \mathrm{l})$ were tested (Supplementary Figs. 8 and 9). With the increase of the water volume from 5 to $50 \mu \mathrm{l}$, the duration of the low COF gradually increased from 430 to $2680 \mathrm{~s}$. The duration decreased from 1300 to $260 \mathrm{~s}$ as the test temperature was increased from 25 to $80^{\circ} \mathrm{C}$. For the oBP nanosheets suspension, the running-in time gradually reduced from about $700 \mathrm{~s}$ at $25^{\circ} \mathrm{C}$ to about $90 \mathrm{~s}$ at $85^{\circ} \mathrm{C}$. At the same time, the superlubricity state was difficult to realize when the test temperature was higher than $65^{\circ} \mathrm{C}$ (Supplementary Fig. 9). It indicates that the difficulty of retaining water molecules at the friction interface is the main challenge for the efficient waterbased lubrication. The excellent water retention property of oBP nanosheets plays an important role in maintaining the low COF. The running-in time reduces from 850 to $100 \mathrm{~s}$ with the increase of the sliding speed from 50 to $250 \mathrm{~mm} / \mathrm{s}$. For the test with the speed of $250 \mathrm{~mm} / \mathrm{s}$, the short-period superlubricity could be achieved even after a running-in time of $50 \mathrm{~s}$ (Fig. 3b).

Figure $3 c$ shows the $3 D$ topographic images of the ball wear scars after tests with different loads and sliding speeds. The actual contact pressure (ACP) in the superlubricity state is estimated by dividing the normal load by the contact area (Fig. 3d). Supplementary Table 1 shows the specific values of the wear scar diameters (WSD), ACPs, and COFs with the lubrication of oBP nanosheets suspensions under various test conditions in the superlubricity state. The wear scar for the oBP nanosheets suspensions are much smaller than those from tests with water, the HP solution and the BP particles suspension, respectively (Supplementary Fig. 7b). The diameter of the wear scar increased from 48 to $86 \mu \mathrm{m}$ with the load increasing from 1 to $4 \mathrm{~N}$, and decreased from 61 to $45 \mu \mathrm{m}$ with the sliding speed increasing from 50 to $250 \mathrm{~mm} / \mathrm{s}$.

It is worth mentioning that the wear scar was almost difficult to measure with the test condition of $2 \mathrm{~N}$ and $250 \mathrm{~mm} / \mathrm{s}$. Accordingly, with the increase of the normal load, the calculated average ACP increased firstly and then decreased, reaching its maximum value of about $1044 \mathrm{MPa}$ under the applied load of $2 \mathrm{~N}$. The variation trend of the ACP is different from that of the contact pressure calculated by Hertzian method (HCP), which increases monotonically from 1148 to $1823 \mathrm{MPa}$ with the load increasing from 1 to $4 \mathrm{~N}$ for the $\mathrm{Si}_{3} \mathrm{~N}_{4}$ /sapphire friction pairs. However, the $\mathrm{HCP}$ can only represent the initial contact pressure during the friction test and the ACP is always lower than the HCP because of the unavoidable wear of the ball surface during the friction test. The higher the normal load, the more serious is the wear of the ball. Therefore, the ACP does not always increase monotonically with the normal load, which is also related to the wear degree of the ball. Although the HCP keeps the same under the same load, the calculated average ACP increased slightly with the increase of the sliding speed (Fig. 3d). The high speed is helpful to form the effective lubricating film, resulting in the reduction of the wear. Importantly, in the case with the test condition of $2 \mathrm{~N}$ and $250 \mathrm{~mm} / \mathrm{s}$, the average final contact pressure for the oBP nanosheets suspension in the superlubricity state could reach $1193 \mathrm{MPa}$, which is the highest contact pressure reported till date for the liquid superlubricity materials (Fig. 3e) ${ }^{18,19,21,22,38-40}$.

It has been seen that with lubrication by $20 \mu \mathrm{l}$ oBP nanosheets suspension, the superlubricity state could keep stable for more than $2 \mathrm{~h}$ (Supplementary Fig. 10a). It can be inferred that the properties of adsorption and retention of water molecules for the $\mathrm{OBP}$ nanosheets give rise to the achievement and sustainment of the superlubricity state. To confirm this hypothesis, the COFs versus time with the lubrication of $20 \mu \mathrm{l}$ suspensions with the oxidized graphene oxide (oGO) nanosheets or the oxidized molybdenum disulfide $\left(\mathrm{oMoS}_{2}\right)$ nanosheets were conducted (Supplementary Fig. 10b). The lubrication state could only sustain for 800 and $650 \mathrm{~s}$ for the oGO and $\mathrm{oMoS}_{2}$ nanosheets suspensions, respectively, owing to water evaporation. The results indicate that the use of oBP nanosheets have great advantages in the achievement of superlubricity, because of the outstanding adsorption and retention abilities of water molecules. The effect of the $\mathrm{H}_{2} \mathrm{O}_{2}$ content on the friction behaviors of oBP nanosheets was also investigated (Supplementary Fig. 10c). Generally, the larger the content of $\mathrm{H}_{2} \mathrm{O}_{2}$, the higher is the oxidation degree of the BP nanosheets. It has been seen that robust superlubricity could be achieved when the ratio of $\mathrm{H}_{2} \mathrm{O}_{2}(\mu \mathrm{l})$ and $\mathrm{BP}(\mathrm{mg})$ is 0.3-4.5. With the excess addition of $\mathrm{H}_{2} \mathrm{O}_{2}$, the superlubricity state is hard to achieve even after a running-in time of $1800 \mathrm{~s}$. It means the structure of the oBP nanosheets is important for the achievement of superlubricity. The friction behaviors with the frictional pairs of $\mathrm{Si}_{3} \mathrm{~N}_{4} / \mathrm{ZrO}_{2}$ ceramic and $\mathrm{Si}_{3} \mathrm{~N}_{4} / \mathrm{SiO}_{2}$ glass were 


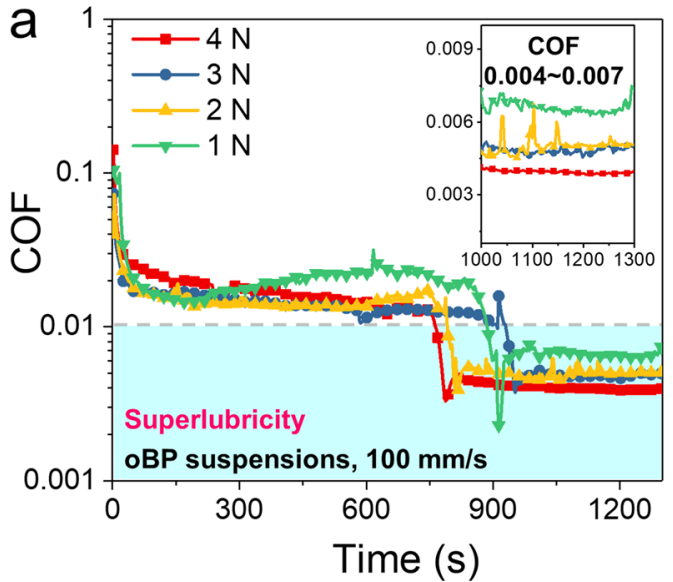

C

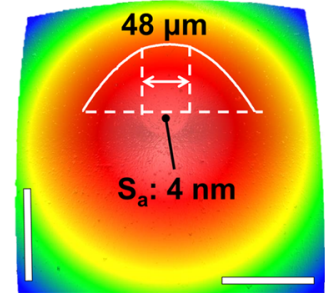

$1 \mathrm{~N}, 100 \mathrm{~mm} / \mathrm{s}$

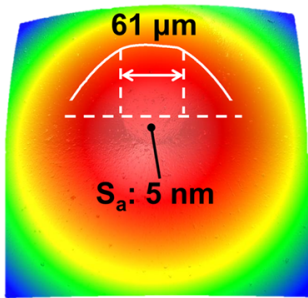

$2 \mathrm{~N}, 50 \mathrm{~mm} / \mathrm{s}$

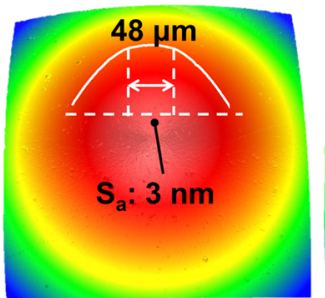

$2 \mathrm{~N}, 100 \mathrm{~mm} / \mathrm{s}$

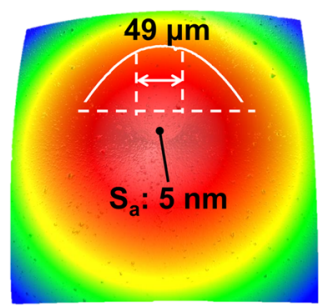

$2 \mathrm{~N}, 150 \mathrm{~mm} / \mathrm{s}$

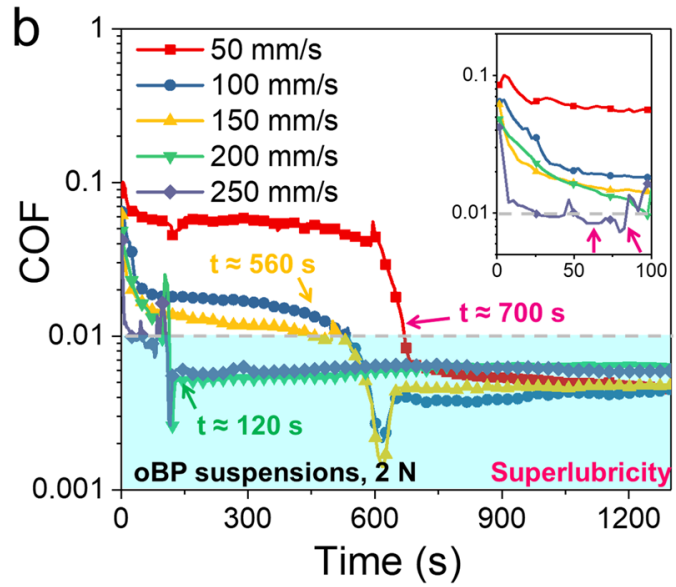

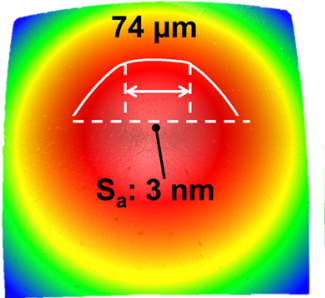

$3 \mathrm{~N}, 100 \mathrm{~mm} / \mathrm{s}$

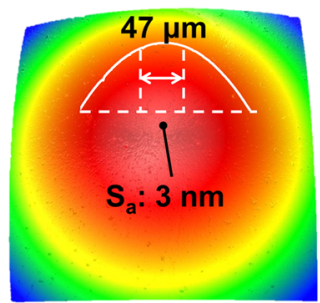

$2 \mathrm{~N}, 200 \mathrm{~mm} / \mathrm{s}$

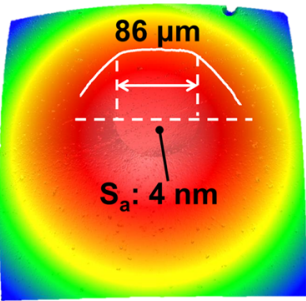

$4 \mathrm{~N}, 100 \mathrm{~mm} / \mathrm{s}$

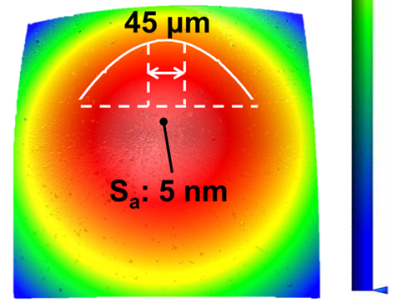

$2 \mathrm{~N}, 250 \mathrm{~mm} / \mathrm{s}$
$4.6 \mu \mathrm{m}$

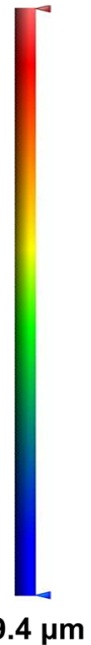

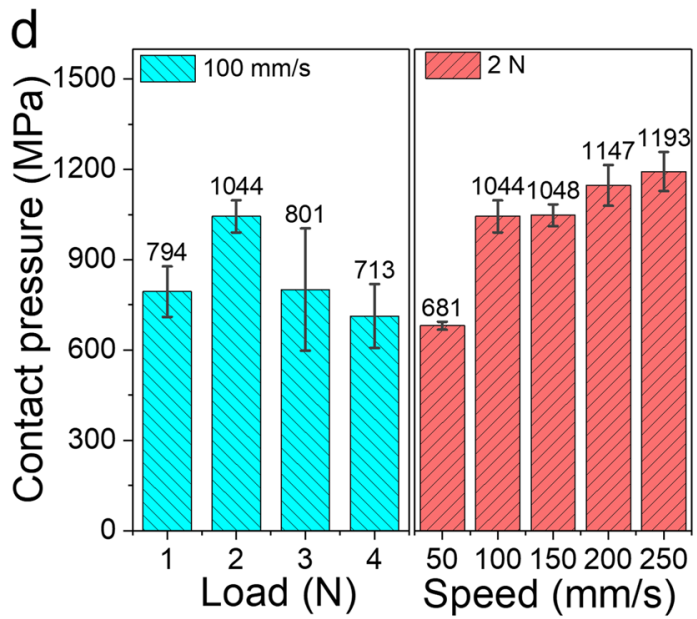

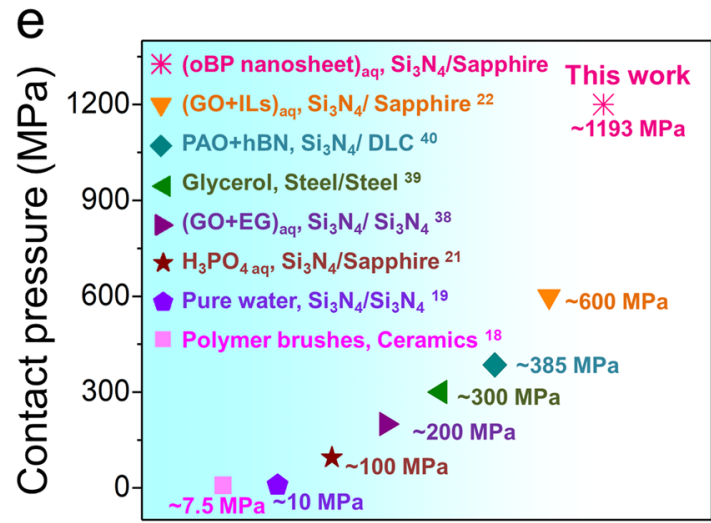

\section{Liquid superlubricity}

Fig. 3 Characterizations of the liquid superlubricity properties of the oBP nanosheets suspensions. a Friction coefficients versus time under various normal loads $(1-4 \mathrm{~N}, 100 \mathrm{~mm} / \mathrm{s})$ and $\mathbf{b}$ with various sliding speeds $(50-250 \mathrm{~mm} / \mathrm{s}, 2 \mathrm{~N})$ of $20 \mu \mathrm{l}$ oBP nanosheets suspensions. c $3 D$ topographic images showing the ball wear scars after testing with different loads and sliding speeds (scale bar: $100 \mu \mathrm{m}$ ). The insets are the profile images of the wear scars. d The corresponding contact pressures in the superlubricity state with various loads and sliding speeds, which were calculated by dividing the normal load by the contact area. e Comparison of the contact pressures of this work with other typical liquid lubrication systems during the liquid superlubricity state ${ }^{18,19,21,22,38-40}$.

also tested to confirm the applicability of the oBP nanosheets suspensions (Supplementary Fig. 10d). For all of the substrates tested, superlubricity could be achieved with the oBP nanosheets suspensions as the lubricants.

\section{Mechanism}

To further clarify the mechanism underlying these friction phenomena, we used surface and interface analytical techniques, such as TEM, Raman, XPS, and EELS. The nanometer-thick lamella 

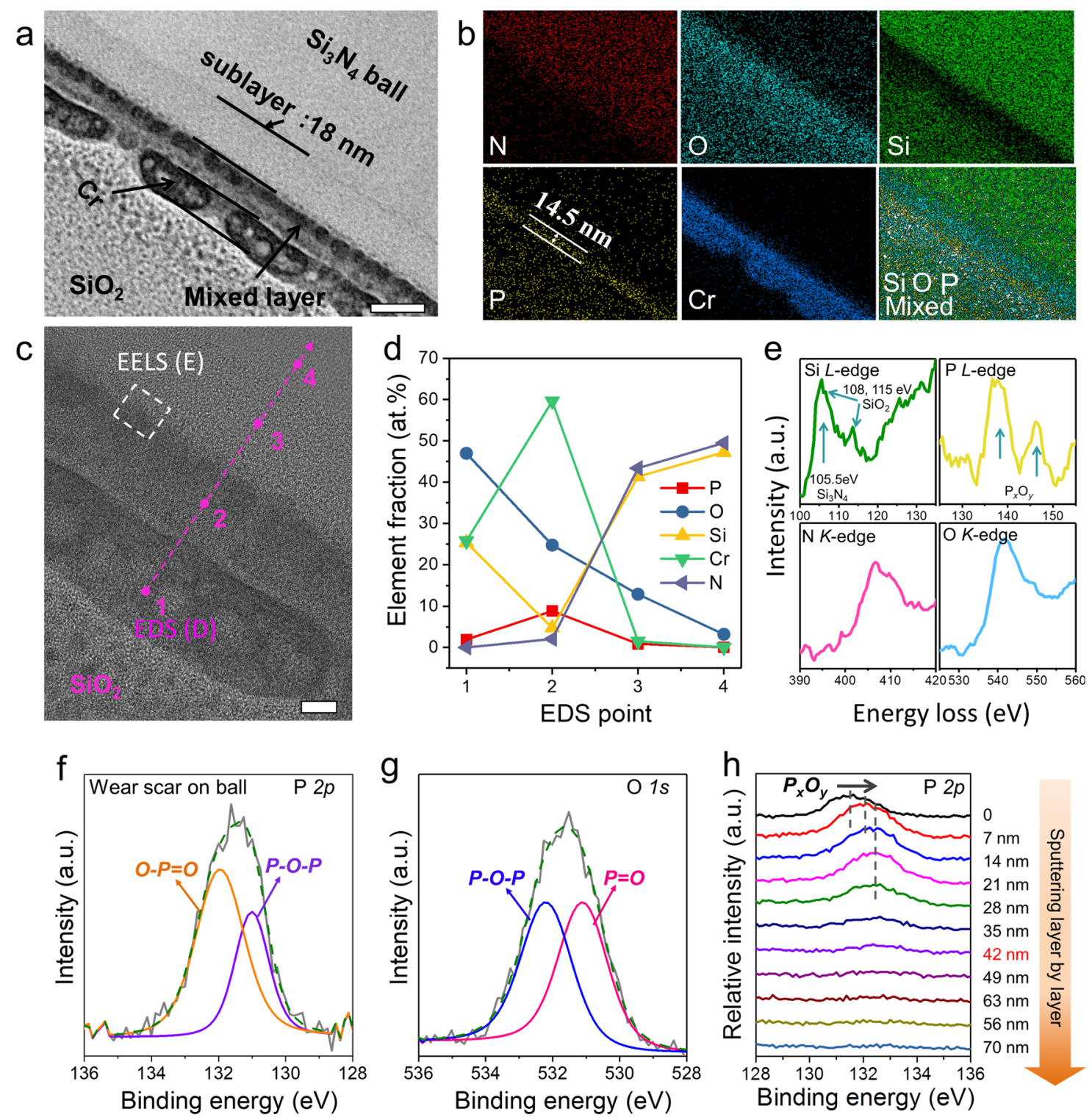

Fig. 4 Interfacial microstructures of the wear scars on the $\mathrm{Si}_{3} \mathbf{N}_{\mathbf{4}}$ ball surfaces after the friction tests. a TEM image showing the interfacial microstructures of the wear scar surface on the $\mathrm{Si}_{3} \mathrm{~N}_{4}$ ball after the friction test with the oBP nanosheets suspension (test condition: $2 \mathrm{~N}$, $100 \mathrm{~mm} / \mathrm{s}$ ). Scale bar: $20 \mathrm{~nm}$. b The corresponding EDS maps, indicating the distributions of P, O, Si, Cr, and N elements. c HRTEM image showing the interfacial microstructures of the wear scar surface after the friction test with the oBP nanosheets suspension. Scale bar: 5 nm. d Evolutions of the fractions (at.\%) of $\mathrm{P}, \mathrm{O}, \mathrm{Si}, \mathrm{Cr}$, and $\mathrm{N}$ elements recorded across the interface as marked in C. e EELS Si- $L$, P- $L, N-K$, and O- $K$ core edge spectra showing the elemental distribution and the bonding structure of the region as marked in c. f, g P $2 \mathrm{p}$ and $\mathrm{O} 1 \mathrm{~s}$ XPS spectra on the wear scar of the $\mathrm{Si}_{3} \mathrm{~N}_{4}$ ball surface after the friction test with the oBP nanosheets suspension. $\mathbf{h} \mathrm{P} 2 \mathrm{p}$ peaks XPS spectra of the wear scar on the $\mathrm{Si}_{3} \mathrm{~N}_{4}$ ball surface obtained by layer-by-layer sputtering.

samples from the wear surfaces on the $\mathrm{Si}_{3} \mathrm{~N}_{4}$ balls and the sapphire discs after the friction tests with the oBP nanosheets suspensions were prepared using the focused-ion beam (FIB) liftout method. The metallic $\mathrm{Cr}$ layer was deposited on the wear surfaces, and a silicon dioxide $\left(\mathrm{SiO}_{2}\right)$ layer or a platinum $(\mathrm{Pt})$ layer was deposited on the FIB slicing position for protection during the ion thinning process. The FIB slicing positions of the selected wear surfaces are indicated in Supplementary Fig. 11.

Figure 4 shows the microstructure and chemical composition of the wear scar surface on the $\mathrm{Si}_{3} \mathrm{~N}_{4}$ ball after the friction test with the oBP nanosheets suspension under the test condition of $2 \mathrm{~N}$ and $100 \mathrm{~mm} / \mathrm{s}$. The interesting finding is the presence of a sublayer (about $18 \mathrm{~nm}$ in thickness) with almost the same contrast but different crystallinities in the high-resolution TEM (HRTEM) images between the sliding surface and the $\mathrm{Si}_{3} \mathrm{~N}_{4}$ ball matrix
(Fig. 4a, c). The EDS mapping indicates the presence of a sublayer enriched with $\mathrm{N}, \mathrm{Si}$, and $\mathrm{O}$ elements (Fig. 4b). This layer demonstrates that a certain amount of $\mathrm{Si}_{3} \mathrm{~N}_{4}$ on the wear surface is oxidized during the friction process. The EDS mapping also shows that a layer mixed with $\mathrm{P}$ and $\mathrm{Cr}$ elements forms on the wear surface during the deposition process of the metallic $\mathrm{Cr}$ layer. It may be pointed out that during the preparation of the FIB samples, the ball was dried under the vacuum condition without cleaning the wear surface after the friction test. A thin porous lubricant film may form during the dry process, and the metallic $\mathrm{Cr}$ diffuses into this layer during the deposition process, resulting in the formation of a mixed layer. In comparison, the microstructure of the cleaned $\mathrm{Si}_{3} \mathrm{~N}_{4}$ ball after the friction test with the oBP nanosheets suspension is shown in Supplementary Fig. 12. The similar sublayer is detected, but the P-containing layer could not 
be found at the interface. The $\mathrm{Cr}$ protection layer was relatively intact and dense. The evolutions of the element fractions (at.\%) in each layer are marked in Fig. 4c, further revealing the existence of the P-rich mixed layer and the O-rich sublayer (compared with the $\mathrm{Si}_{3} \mathrm{~N}_{4}$ matrix) (Fig. $4 \mathrm{~d}$ and Supplementary Table 2).

To quantitatively clarify the bonding structure of the mixed layer and the sublayer, EELS core edge spectra were recorded in the area as marked in Fig. 4c. The Si L-edge spectrum confirmed the existence of $\mathrm{Si}_{3} \mathrm{~N}_{4}(105.5 \mathrm{eV})$ and $\mathrm{SiO}_{2}(108$ and $115 \mathrm{eV})$ at the interface. At the same time, the $\mathrm{P} L$-edge spectrum indicates that $\mathrm{P}$ is mainly in the form of phosphorus oxide (138 and $146 \mathrm{eV}$ ). These findings explicitly demonstrate the formation of $\mathrm{SiO}_{2}$ in the sublayer and the presence of $\mathrm{P}_{\mathrm{x}} \mathrm{O}_{\mathrm{y}}$ on the wear scar surface after the friction test with the oBP nanosheets suspension. The $P 2 p$ and $O$ 1s XPS narrow spectra further confirm the existence of the $P$ element on the surface of the wear scar. The peaks detected at 131.8 and $132.75 \mathrm{eV}$ were assigned as the bridging (P-O-P) and dangling $(\mathrm{O}-\mathrm{P}=\mathrm{O})$ phosphorus-oxygen bonds, respectively (Fig. $4 f^{23,29,41,42}$. The bridging oxygen penetrates into the lattice, pushes the neighboring phosphorus atoms outward to form a bridging bond between two phosphorus atoms, giving rise to the dramatic lattice deformation. The dangling oxygen changes the surface affinity and serves as the anchors for hydrogen bonds with water molecules. In addition, $\mathrm{P}=\mathrm{O}$ bonds are reported to have an inclination of forming two hydrogen bonds immediately, enhancing the adsorption of water molecules onto the oBP nanosheets ${ }^{23}$. Owing to the massive $\mathrm{P}=\mathrm{O}$ bonds, water layers would form through hydrogen bonds, transforming the shear interface from the BP/BP interface to the retained water molecules layer, which are helpful to the achievement of superlubricity. The peak intensity ratio of $\mathrm{P}=\mathrm{O}$ to $\mathrm{P}-\mathrm{O}-\mathrm{P}$ was about 1.8 , which is much higher than their relative population (2:3) in the $\mathrm{P}_{2} \mathrm{O}_{5}$ structure. It is reported that termination with $\mathrm{P}=\mathrm{O}$ (dangling oxygens) could provide a protection layer with the underlying $\mathrm{BP}^{4 \uparrow}$. The uniform surface oxide layer is likely to affect the photoinduced charge transfer from $\mathrm{BP}$ to aqueous $\mathrm{O}_{2}$, which is the rate-determining step of the ambient oxidation ${ }^{41}$. The $O 1 \mathrm{~s}$ scan shows the peaks at 530.7 and $532.3 \mathrm{eV}$ on the wear surface of the ball, assigned as the $\mathrm{P}=\mathrm{O}$ and $\mathrm{P}-\mathrm{O}-\mathrm{P}$ bonds (Fig. $4 \mathrm{~g})^{23,42}$. The sputtering layer-by-layer XPS spectra of $P 2 p$ are shown in Fig. $4 \mathrm{~h}$. It can be seen that the film thickness with phosphorus is about $42 \mathrm{~nm}$. The position of the P $2 p$ peak gradually shifts to the higher binding energy direction, indicating that the oxidation extent of the deeper layer is higher than that of the surface layer ${ }^{23}$.

The superlubricity behavior achieved by phosphoric acids between the $\mathrm{Si}_{3} \mathrm{~N}_{4}$ ball and the sapphire disc was observed in previous work ${ }^{21}$. However, the contact pressure was rarely higher than $300 \mathrm{MPa}$ during the superlubricity state. It was supposed that the oBP nanosheets rather than the oxygenic acid of phosphorus (formed by the $\mathrm{P}_{\mathrm{x}} \mathrm{O}_{\mathrm{y}}$ solving in water) played the main role for the achievement of superlubricity at the extremely high-contact pressure. The speculation was evidenced by the characterization of the wear trace on the sapphire disc. The optical images show that about $900 \mu \mathrm{m}$ wide residual liquid trace and $75 \mu \mathrm{m}$ actual contact trace can be observed on the wear trace after the friction test with the oBP nanosheets suspension (Fig. 5a, b). A lot of black flakes and gray flakes can be observed in the liquid trace (Fig. 5a) and the contact trace (Fig. 5b), respectively. The Raman spectra of these flakes (Fig. $5 \mathrm{C}$ ) confirm that they are both the aggregation of the oBP nanosheets, owing to the presence of characteristic BP peaks, $A_{g}{ }^{1}, B_{2 g}$, and $A_{g}{ }^{2}$ modes ${ }^{23}$. The gray flakes are much thinner than the black ones, resulting in the detection of the sapphire matrix in the Raman spectra. Indeed, the Raman intensities of the gray flakes are much lower (one-fifth) than those of the black ones, and the peak position of $\mathrm{A}_{\mathrm{g}}{ }^{2}$ is shifted to the higher wavenumber, both of which demonstrate that the gray flakes are thinner than the black ones ${ }^{23}$. Meanwhile, the degree of oxidization of the gray flakes is much higher than that of the black ones, which is confirmed by the intensity differences of the Raman peaks at $900 \mathrm{~cm}^{-1}$ (assigned to the $\left.\mathrm{P}_{\mathrm{x}} \mathrm{O}_{\mathrm{y}}\right)^{29}$.

Interestingly, the thin gray oBP flakes are mainly distributed in the contact trace, while the black ones stay outside the contact area. More vividly shown in the spatial Raman map $\left(A_{g}{ }^{2}\right.$ intensity, Fig. $5 \mathrm{~d}$ ), the contact trace on the wear track of the sapphire disc is rich in oBP nanosheets. Single spectra extracted at the positions marked in Fig. $5 \mathrm{~d}$ are displayed in Fig. 5e. It may be noted that the wide and weak peaks at $880-920 \mathrm{~cm}^{-1}$ are mainly assigned to the Raman active modes of phosphorus oxides $\left(\mathrm{P}_{\mathrm{x}} \mathrm{O}_{\mathrm{y}}\right)$, and detected at all the points, indicating the widespread existence of the $\mathrm{P}_{\mathrm{x}} \mathrm{O}_{\mathrm{y}}$ film in the contact track after the friction test. The peaks at 381,418 , and $645 \mathrm{~cm}^{-1}$ are assigned to the Raman modes of sapphire. These findings indicate that the thinner oBP nanosheets with the higher degree of oxidation are likely to be dragged into the friction interface. In consideration of the excellent ability of the adsorption and retention of water molecules at the oBP nanosheet surfaces, the oBP nanosheets act as the water-rich lubricant additives and retain water at the friction interface even under ultrahigh contact pressures.

As revealed by the high-angle annular dark-field-STEM images in Fig. $5 f, g$, the as-formed lubricant film after vacuum drying on the disc wear track surface is very thick $(\sim 260 \mathrm{~nm})$ and porous. The relevant bonding states of the lubricant film are further analyzed by EELS. As shown in Fig. 5h, the EELS spectrum images of the elemental maps of $\mathrm{P}-L, \mathrm{Al}-L$, and $\mathrm{O}-K$ indicate that phosphorus and oxygen are distributed throughout the lubricant film. Aluminum is only detected on the sapphire surface, indicating that no sapphire debris entered into the lubrication film. The result is consistent with the fact that the wear loss on the sapphire was almost unmeasurable. To further clarify the bonding structure of the lubricant film and the interface, the EELS SI point profiles of $P L_{2,3^{-}}$ edge were recorded point by point in the lubricant film, as shown in Fig. $5 \mathrm{~g}$. The phosphorus $L$-edge could be used to identify the chemical valence state ${ }^{24,43}$. In point 1 , no peak of $P$ L-edge could be seen, which is consistent with the matrix elemental composition. In point 2 , the intensity of the $P$ L-edge peak is very weak, indicating that the black region is the pore in the lubricant film. In points $3-7$, both the BP signals $(131 \mathrm{eV})$ and the $\mathrm{P}_{\mathrm{x}} \mathrm{O}_{\mathrm{y}}$ signals (138 and $146 \mathrm{eV}$ ) are detected. The finding once again confirms the presence of oBP nanosheets at the friction interface, and the BP structure remained after the friction test.

The MD simulations are performed to further explore the superlubricity mechanism under the ultrahigh contract pressure (Fig. 6a-d). It is reported that the nano-friction behavior of phosphorene and few-layer BP flakes exhibits anisotropic effects due to the structural anisotropy ${ }^{44-46}$. Layers stacked with the armchair-zigzag orientation shows a remarkable superlubricity, with a reduction of one order of magnitude for the shear stress based on the first-principles calculations ${ }^{46}$. However, the interface conditions of oBP nanosheets are different from phosphorene or few-layer BP flakes. Motivated by our experimental analyses, five models of the friction interfaces, including the BP/BP, BP-O/BP-O, $\mathrm{BP}-\mathrm{OH} / \mathrm{BP}-\mathrm{OH}, \mathrm{SiO}_{2} / \mathrm{BP}-\mathrm{O}$, and $\mathrm{SiO}_{2} / \mathrm{BP}-\mathrm{OH}$, are studied (Supplementary Fig. 14). In these models, the $\mathrm{BP}-\mathrm{O}$ and $\mathrm{BP}-\mathrm{OH}$ correspond to the $\mathrm{P}=\mathrm{O}$ and $\mathrm{P}-\mathrm{OH}$ bonds, respectively, which are formed on the $\mathrm{OBP}$ nanosheet surfaces during the oxidation process. In consideration of the formation of $\mathrm{SiO}_{2}$ on the wear scar, the friction interfaces contained $\mathrm{SiO}_{2}$ are also studied. The effect of the adsorbed water molecules on the friction behavior is analyzed by filling single-layer and three-layer water molecules at the friction interfaces of the models. It is reported that intercalated water molecules would hinder the sliding motion of both $\mathrm{MoS}_{2}$ layers and graphene layers based on the ab initio MD calculation ${ }^{47,48}$. Interestingly, this phenomenon is in contrast to the lubricating functionality of intercalated water molecules in oBP nanosheets. With the water layer at the friction interfaces, the interface pressures at the friction interfaces of the BP-O/BP-O, BP-OH/BP- 

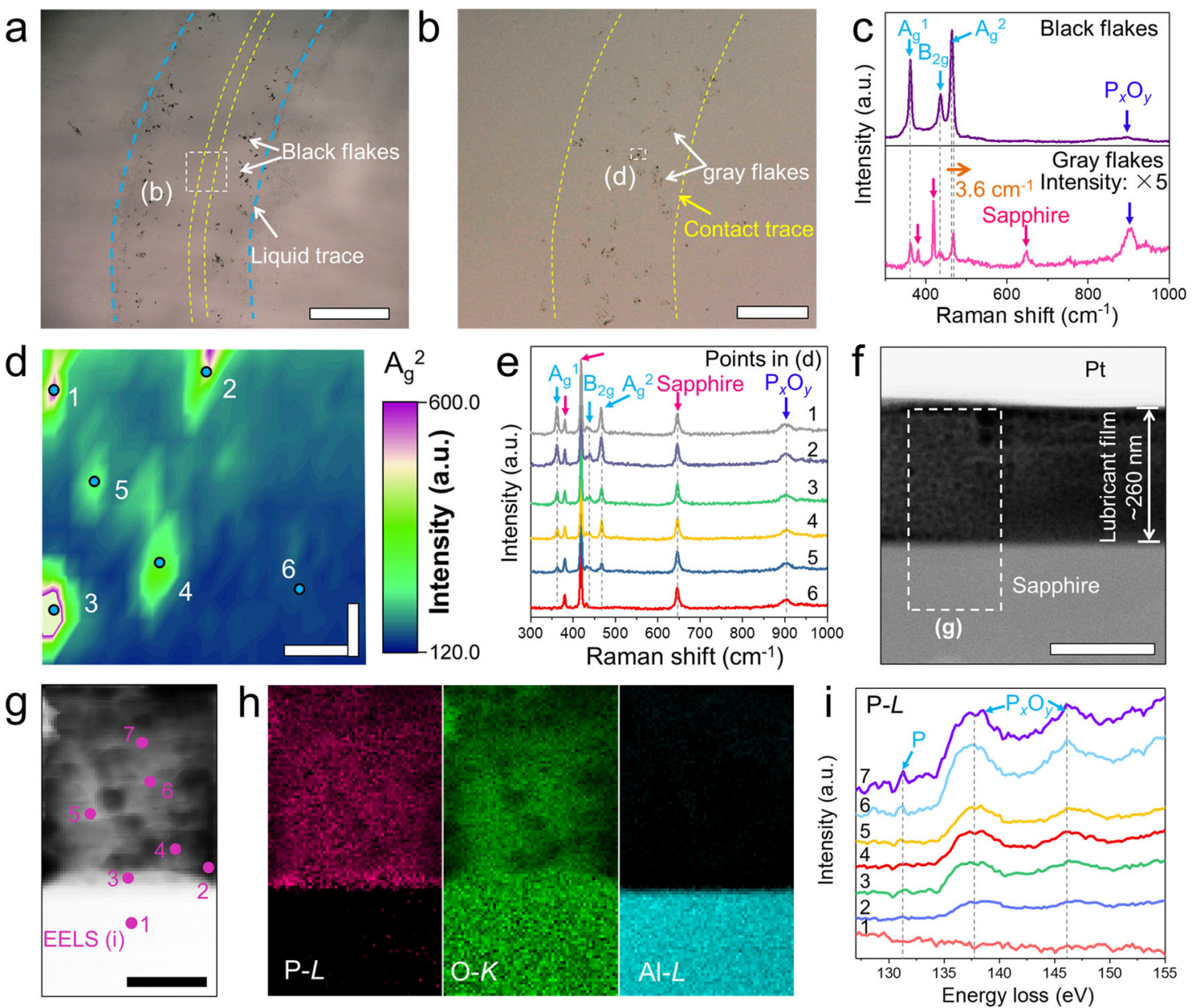

Fig. 5 Characterization of the wear trace on the sapphire disc surface after the friction test with the oBP nanosheets suspension (test condition: $\mathbf{2} \mathbf{N}$ and $100 \mathrm{~mm} / \mathrm{s}$ ). a Optical image showing the residual liquid traces after the friction test. Scale bar: $500 \mu \mathrm{m}$. b Optical image showing the actual contact trace with the wear scar on the $\mathrm{Si}_{3} \mathrm{~N}_{4}$ ball as marked in a. Scale bar: $50 \mu \mathrm{m}$. c Raman spectra of the black and gray flakes as marked in $\mathbf{a}, \mathbf{b}$. d Raman $\mathrm{A}_{\mathrm{g}}{ }^{2}$ intensity map of the region as marked in $\mathbf{b}$. Scale bar: $2 \mu \mathrm{m}$. e Raman spectra of the region as marked in d. $\mathbf{f}, \mathbf{g}$ HAADF-STEM images showing the existence of a porous lubricant film on the top of the sapphire disc surface. Scale bar: $200 \mathrm{~nm}$ in $\mathbf{f}$ and $100 \mathrm{~nm}$ in $\mathbf{g}$. The position for the EELS spectrum image mapping and the acquisition points are also indicated. $\mathbf{h}$ EELS P-L, O- $K$, and Al- $L$ maps showing the elemental distributions in the region as marked in $\mathbf{f}, \mathbf{g}$. i Evolutions of EELS P-L core edge spectra recorded at the points as marked in $\mathbf{g}$.

$\mathrm{OH}, \mathrm{SiO}_{2} / \mathrm{BP}-\mathrm{O}$, and $\mathrm{SiO}_{2} / \mathrm{BP}-\mathrm{OH}$ are all lower than that at the friction interfaces of the BP/BP (Fig. $6 \mathrm{c}$, d). The water molecules could be kept on the BP-O or BP-OH surfaces by the hydrogen bonds even under the ultrahigh contact pressure in the simulations. A superlubricity state with a COF of 0.0026 is achieved at the $\mathrm{SiO}_{2} / \mathrm{BP}-\mathrm{OH}$ friction interface with the adsorption of single-layer water molecules. The trajectories of the layers indicate that the sliding process occurs between the $\mathrm{SiO}_{2}$ and the adsorbed water layer at the $\mathrm{SiO}_{2} / \mathrm{BP}-\mathrm{OH}$ friction interface (Supplementary Fig. 15).

However, the COFs at the friction interfaces of the BP-O/BP-O and $\mathrm{BP}-\mathrm{OH} / \mathrm{BP}-\mathrm{OH}$ with single-layer water molecules are even higher than that without water molecules (Supplementary Fig. 16). The trajectories of the layers indicate that the strong hydrogen bonds between the water molecules and the BP-O/BP-O or BP-OH/ $\mathrm{BP}-\mathrm{OH}$ interfaces make the single-layer water glue the upper and lower interfaces, resulting in the higher COFs. Yet, with three-layer water molecules between the interfaces, the COFs are much lower than that without the adsorption of water molecules. A superlubricity state with a COF of 0.0017 is also achieved at the friction interface of $\mathrm{BP}-\mathrm{OH} / \mathrm{BP}-\mathrm{OH}$ (Fig. 6d). The trajectories suggest that the middle one in the three-layer water molecules is prone to slipping because of the weak hydrogen bonds with the friction interface. The sliding occurs mainly at the middle layer water, resulting in the superlow $\mathrm{COF}$ at the $\mathrm{BP}-\mathrm{OH}$ interface with threelayer water molecules (Supplementary Fig. 17). The simulated results of the interface pressure, friction force, and COF also indicate that the interface pressures at the friction interfaces with the water molecules in the interlamination are relatively lower than that without the water molecules (Supplementary Fig. 18). The reduction of the interface pressures is helpful to achieve the low COF even under the high-contact pressure.

According to the above analyses, the schematic illustration of the superlubricity mechanism under the ultrahigh contact pressure with the lubrication of the oBP nanosheets suspension has been proposed, as shown in Fig. 6e, f. Two aspects have been attributed to the superlubricity under the ultrahigh contact pressure: firstly, oBP nanosheets adsorbed on the contacting surface, transforming the shear interface from $\mathrm{Si}_{3} \mathrm{~N}_{4}$ /sapphire interface into the oBP/oBP nanosheets interface; and secondly, the oBP nanosheets adsorbed water molecules to form a retained water layer, decreasing the COF. During the running-in period, 
a

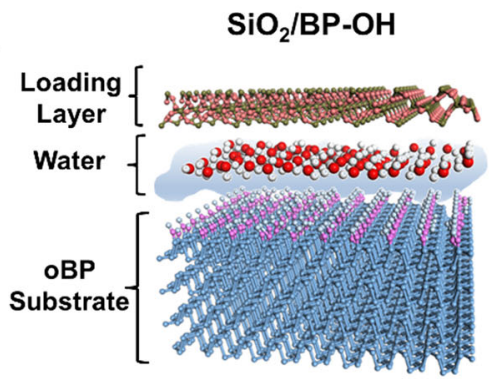

C

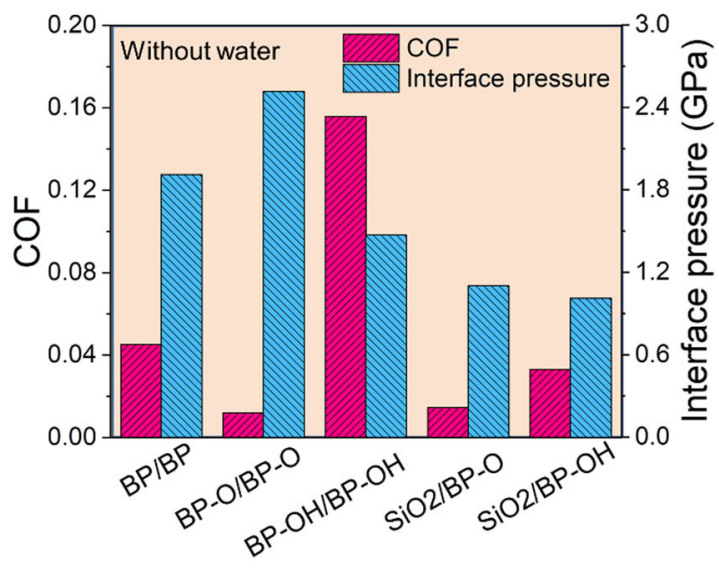

e

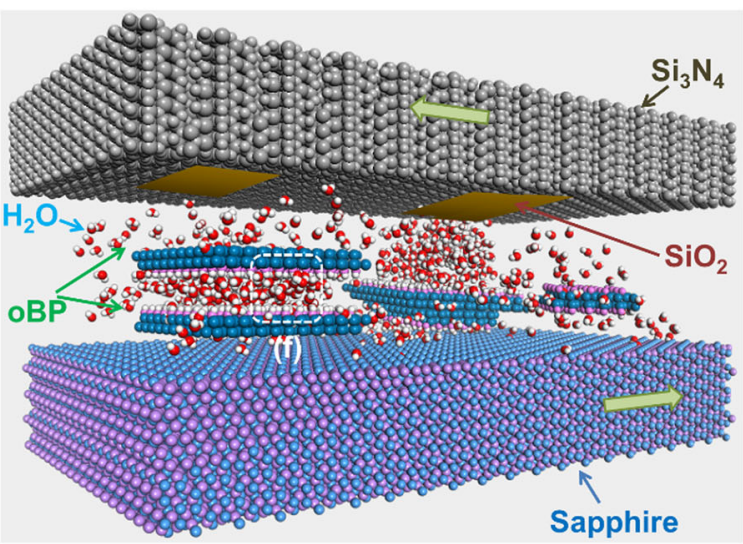

BP-OH/BP-OH

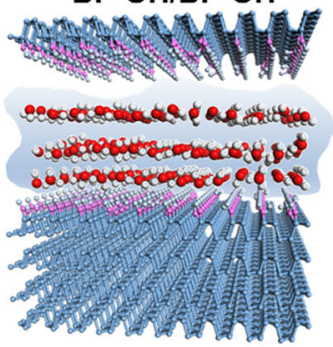

d

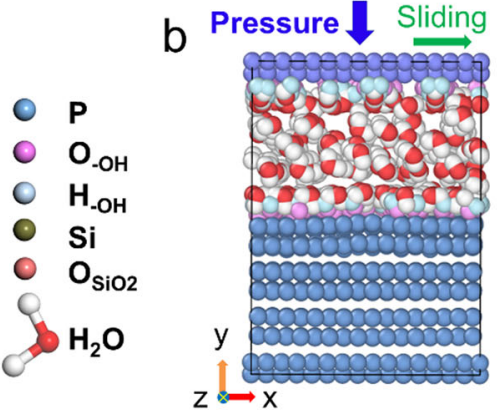

$z \stackrel{x}{x}$
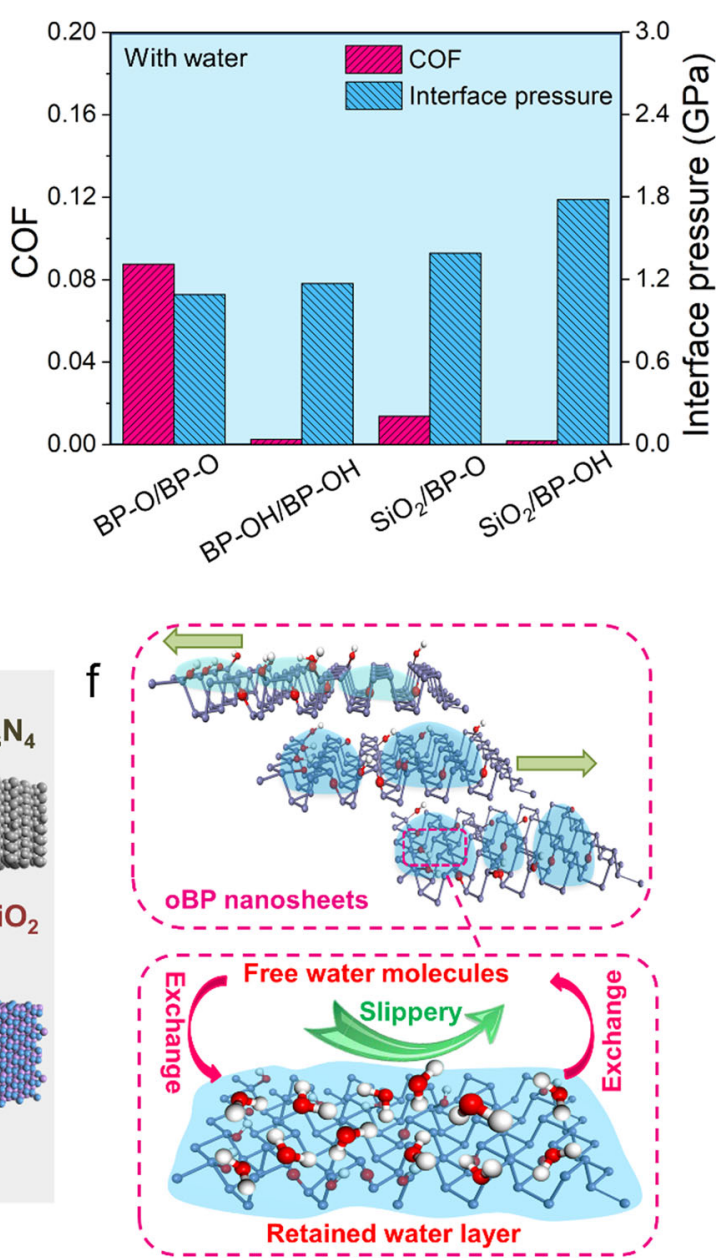

Fig. 6 Molecular dynamics (MD) simulations of the sliding processes at the $\mathrm{SiO}_{2} / \mathrm{oBP}$ and oBP/oBP interfaces. a Modeled structures of the $\mathrm{SiO}_{2} / \mathrm{oBP}$ and oBP/oBP systems. b Typical simulated LAMMPS model. Variations of the interface pressures and COFs under an applied pressure of $1 \mathrm{GPa}$ pressure and a speed of $1 \mathrm{~m} / \mathrm{s}$ without (c) and with (d) water molecules involved at the friction interface. Five groups at the friction interface, including $\mathrm{BP} / \mathrm{BP}, \mathrm{BP}-\mathrm{O} / \mathrm{BP}-\mathrm{O}, \mathrm{BP}-\mathrm{OH} / \mathrm{BP}-\mathrm{OH}, \mathrm{SiO}_{2} / \mathrm{BP}-\mathrm{O}$, and $\mathrm{SiO}_{2} / \mathrm{BP}-\mathrm{OH}$, have been studied. For water involved at the friction interfaces, the single-layer water molecules are filled for $\mathrm{SiO}_{2} / \mathrm{BP}-\mathrm{O}$ and $\mathrm{SiO}_{2} / \mathrm{BP}-\mathrm{OH}$, and three-layer water molecules are filled for $\mathrm{BP}-\mathrm{O} / \mathrm{BP}-\mathrm{O}$ and $\mathrm{BP}-\mathrm{OH} / \mathrm{BP}-\mathrm{OH}$. e, $\mathbf{f}$ Schematic illustration of the proposed lubrication model in this work.

some oBP nanosheets enter into the contact interface, and a thin boundary layer with the oBP nanosheets is formed on the wear track. Due to the transformation of the shear interface from $\mathrm{Si}_{3} \mathrm{~N}_{4} /$ sapphire into the $\mathrm{OBP} / \mathrm{OBP}$, which adsorbs plenty of water molecules near their surfaces, the COF is much lower than that without the oBP nanosheets addition. The formation of the oBP nanosheets layer at the interface is also helpful in reducing the wear of the friction pairs, resulting in negligible wear of the friction pairs. The $\mathrm{OBP}$ nanosheets at the interface are similar to the extreme pressure additives with plenty of water molecules adsorbed on their surfaces. The BP crystal structure in the oBP nanosheets provides the extremely high supporting capacity, and the adsorbed water layer gives the superlow friction coefficient. The extreme pressure property of the BP nanosheets allows them to be trapped in the contact zone, forming an adsorption layer. The boundary layer formed by OBP nanosheets contributes to the achievement of superlubricity under the ultrahigh pressure state, because of the low wear during the running-in period and the low shear stress between the oBP interlayers and the retained water layers. 
In summary, partially oxidized BP nanosheets aqueous suspensions have been used as the lubricants to achieve a robust superlubricity state $(\mu \approx 0.004)$ under an ultrahigh contact pressure of $1193 \mathrm{MPa}$ at the $\mathrm{Si}_{3} \mathrm{~N}_{4}$ /sapphire interface, which significantly enhance the upper limit of the contact pressure in liquid superlubricity. The oBP nanosheets trapped in the contact zone during sliding result in the transformation of the shear interface from $\mathrm{Si}_{3} \mathrm{~N}_{4} /$ sapphire into the oBP/oBP nanosheets. MD simulations demonstrate that the $\mathrm{P}=\mathrm{O}$ and $\mathrm{P}-\mathrm{OH}$ bonds formed during oxidation could adsorb and retain water molecules at the oBP surface, contributing to the achievement and persistence of superlubricity under the ultrahigh contact pressure. The present work suggests a simple and efficient method for the design of high-performance superlubricity materials and the acceleration of their realizations in industrial applications, such as the lubrication of underwater vehicles and the water-based rolling fluid.

\section{METHODS}

\section{Synthesis of partially oxidized BP nanosheets}

The BP powders were prepared by the high-energy ball-milling technique using RP (purityå 99\%, Aladdin Ltd) as the starting materials. For the oxidation and exfoliation of BP from bulk to the porous layer structure, the dilute HP solution was used as the oxidizing agent, and the sonication technique was applied. Firstly, 50, 250, 500, and $750 \mu \mathrm{l} \mathrm{HP}$ aqueous solutions (30 wt\% of $\mathrm{H}_{2} \mathrm{O}_{2}$, Aladdin Ltd) were added to $10 \mathrm{ml}$ deionized water, respectively, and then stirred for $10 \mathrm{~min}$. Secondly, $50 \mathrm{mg}$ BP powder was added into the solution and stirred for another $30 \mathrm{~min}$. Subsequently, the mixture was sonicated for $2 \mathrm{~h}$ in the ice-bath. After the sonication, the dispersion was centrifuged at $2000 \mathrm{rpm}$ for $20 \mathrm{~min}$ to remove the large particles. The supernatant was the partially $\mathrm{OBP}$ nanosheets suspension. The ratio of the $\mathrm{H}_{2} \mathrm{O}_{2}(\mu \mathrm{l})$ and $\mathrm{BP}(\mathrm{mg})$ was designed as $0.3,1.5,3$, and 4.5 . As control, the samples without the addition of HP and with excessive amounts of HP were also prepared with the same method. Using GO (Aladdin Ltd) and molybdenum disulfide $\left(\mathrm{MoS}_{2}\right.$, Aladdin Ltd) powder as the raw materials, the oGO, and oxidized $\mathrm{MoS}_{2}\left(\mathrm{OMoS}_{2}\right)$ were also prepared with similar method. The ratio of the $\mathrm{H}_{2} \mathrm{O}_{2}(\mu \mathrm{l})$ and $\mathrm{GO}$ or $\mathrm{MoS}_{2}(\mathrm{mg})$ was designed as 1.5.

\section{Characterization of OBP nanosheets}

The topographies of the oBP nanosheets were obtained by AFM (Cypher S, Asylum Research), SEM (Quanta 200 FEG, FEI), and HRTEM (JEM2010, JEOL). The chemical groups on the OBP nanosheet surfaces were characterized by XPS (PHI Quantera II, Ulvac-Phi Inc). The crystal structures of the prepared BP powder were characterized by XRD (D8-Advance, Bruker) within the $2 \theta$ range of $10-80^{\circ}$. The TGA of the oBP nanosheets was carried out using a TGA Q5000 system with the platinum-HT pan. The samples were heated under flowing nitrogen gas $(50 \mathrm{ml} / \mathrm{min})$ from 25 to $600^{\circ} \mathrm{C}$ with a temperature ramp rate of $10^{\circ} \mathrm{C} / \mathrm{min}$. The initial BP powder and $\mathrm{BP}$ powder oxidized only with water (BP-water) were also characterized as a control. The chemical groups and interactions of oBP nanosheets were detected by FTIR spectroscopy (Spotlight 400, PerkinElmer) and Raman spectroscopy (HR Evolution, Horiba), respectively.

\section{Superlubricity experiments}

The friction experiments were conducted by a universal micro-tribotester (UMT-3, Bruker) in the rotation mode under ambient conditions. The temperature was $26 \pm 2{ }^{\circ} \mathrm{C}$ with a relative humidity of $40-55 \% . \mathrm{Si}_{3} \mathrm{~N}_{4}$ balls ( $4 \mathrm{~mm}$ in diameter and roughness $R_{a}$ of about $10 \mathrm{~nm}$ ) and sapphire discs ( $R_{a}$ of about $1 \mathrm{~nm}$ ) were used as the friction pairs. Prior to the tests, the balls and discs were all sequentially cleaned in ethanol, acetone, and pure water (20 min for each reagent). During the tests, the oBP nanosheets suspensions were introduced into the contact surfaces with a volume of $20 \mu \mathrm{l}$. The applied load on the disc varied from 1 to $4 \mathrm{~N}$, corresponding to an initial contact pressure of $1.15-1.82 \mathrm{GPa}$ at the interface according to the Hertz contact calculation. The sliding velocity ranged from 50 to $250 \mathrm{~mm} / \mathrm{s}$. Each lubricant was tested at least three times to ensure the reproducibility. The experimental error was minimized as much as possible by adjusting the tribotester platform until the same COFs were acquired for clockwise and counterclockwise rotations.

\section{Surface characterization}

The WSD and $R_{a}$ of the wear surfaces on each ball and disc were measured using a 3D white-light interferometer (Nexview, ZYGO Lamda). The chemical groups and interactions of the wear scars on the balls and the wear tracks of the discs were analyzed by XPS (PHI Quantera II, Ulvac-Phi Inc) and Raman spectroscopy (HR Evolution, Horiba) with a laser wavelength of $532 \mathrm{~nm}$. The nanoscale thick lamellar specimens for TEM, STEM, and EELS observations were prepared by a dual-beam SEM/FIB system (FEI Quanta 3D FEG) using an in situ lift-out technique. Field-emission HRTEM (JEOL 2100F) and cold field-emission gun STEM (Titan 80-30) equipped with a Gatan Quantum EELS spectrometer were used to characterize the FIB lamellar specimens. The acceleration voltage was $300 \mathrm{kV}$.

\section{Molecular dynamics simulations}

MD simulations are performed with the large-scale atomic/molecular massively parallel simulator ${ }^{49}$ (http://lammps.sandia.gov) to mimic the sliding processes at the BP/BP, BP-O/BP-O, BP-OH/BP-OH, $\mathrm{SiO}_{2} / \mathrm{BP}-\mathrm{O}$, and $\mathrm{SiO}_{2} / \mathrm{BP}-\mathrm{OH}$ friction interfaces. In these models, $\mathrm{BP}-\mathrm{O}$ and $\mathrm{BP}-\mathrm{OH}$ were used to mimic the $O B P$ nanosheets with the $\mathrm{P}=\mathrm{O}$ and $\mathrm{P}-\mathrm{OH}$ bonds, which formed during the oxidation process. The applied oxidation and hydroxylation active sites on the BP surfaces were thermodynamic stable according to our previous study, where $50 \%$ atoms at the interface were the phosphorus atoms ${ }^{29}$. The $\mathrm{SiO}_{2}$ layer was used to study the effect of $\mathrm{SiO}_{2}$ (formed on the wear scar of the ball) on the friction behavior. A single upper layer was modeled to sustain the externally applied pressures and the tangential speed. Four-layer of the $B P$ with or without the $P=O$ and $\mathrm{P}-\mathrm{OH}$ bonds on the surfaces were modeled to mimic the substrate. The effect of the adsorbed water molecules on the friction behavior was analyzed by filling single-layer (about 100 water molecules) and three-layer (about 300 water molecules) free water molecules at the friction interfaces of the models. The size of the model unit cell is $2.5 \mathrm{~nm} \times 3.7 \mathrm{~nm} \times 3.5 \mathrm{~nm}$ in the $x, y$, and $z$ directions, respectively. The boundary conditions of the unit cell are periodic along the $x$ and $z$ directions, and shrink-wrapped along the $y$ direction.

The interactions within the BP layers were modeled by using the Stillinger-Weber potential. The atomic interactions between the oxygen or hydroxyl groups and the phosphorous atoms are described by the universal force field (UFF) potential ${ }^{50}$. The UFF parameters are summarized in Supplementary Table 3. The atomic interactions of water molecules were modeled by using the simple point charge potential $\left.\right|^{51}$. The hydrogen bonds between water molecules and the oxygen atom in BP-O or the hydroxyl group in BP-OH are described by fitting the first-principal calculation results into the 12-6 Lennard-Jones (L-J) potential model ${ }^{30}$. The other interactions of water molecules with the phosphorous atoms, with the $\mathrm{O}$ atom in the BP-O, and with the hydroxyl groups of $\mathrm{BP}-\mathrm{OH}$ are described by the van der Waals interactions by using the typical 12-6 L-J potential. The L-J parameters were listed in Supplementary Table 3.

The modeled configurations were firstly performed with a minimization calculation until the forces converging to $10^{-16} \mathrm{eV} / \AA$. The obtained configurations were subsequently subjected to a microcanonical ensemble (NVE) MD simulation for $120 \mathrm{ps,} \mathrm{where} \mathrm{the} \mathrm{sustainable} \mathrm{single} \mathrm{layer} \mathrm{was}$ fixed along the $x$ and $z$ directions, and the bottom layer of the BP were fully fixed. The atoms in the middle layer of the substrate in the configuration were allowed to evolve freely with Langevin thermostat at $300 \mathrm{~K}$. The velocities of water molecules were initialized at $300 \mathrm{~K}$ and allowed to evolve freely for further simulations. After the configurations were fully relaxed, five different normal applied pressures $(0.1,0.5,1.0,1.5$, and $2.0 \mathrm{GPa}$ ) were applied on the sustainable single layer to mimic the loading process. Another $400 \mathrm{ps}$ MD simulations were performed for the configuration of single-layer water molecules configurations, and $800 \mathrm{ps}$ MD simulations for the configuration of three-layer water molecules. In the cases of the loaded configurations, the velocity of 1 or $5 \mathrm{~m} / \mathrm{s}$ along the $x$ direction was applied on the sustainable layer to mimic the sliding process.

\section{DATA AVAILABILITY}

All the data needed to evaluate the conclusions in the paper are present in the paper and/or the Supplementary materials. Additional data related to this paper may be requested from the authors.

Received: 15 October 2020; Accepted: 24 February 2021; Published online: 16 April 2021 


\section{REFERENCES}

1. Hirano, M. Superlubricity: a state of vanishing friction. Wear 254, 932-940 (2003).

2. Baykara, M. Z., Vazirisereshk, M. R. \& Martini, A. Emerging superlubricity: a review of the state of the art and perspectives on future research. Appl. Phys. Rev. 5, 041102 (2018).

3. Holmberg, K. \& Erdemir, A. Influence of tribology on global energy consumption, costs and emissions. Friction 5, 263-284 (2017).

4. Martin, J. M., Donnet, C., Lemogne, T. \& Epicier, T. Superlubricity of molybdenumdisulfide. Phys. Rev. B 48, 10583-10586 (1993).

5. Li, H. et al. Superlubricity between $\mathrm{MoS}_{2}$ monolayers. Adv. Mater. 29, 1701474 (2017).

6. Dienwiebel, M. et al. Superlubricity of graphite. Phys. Rev. Lett. 92, 126101 (2004).

7. Zhang, R. et al. Superlubricity in centimetres-long double-walled carbon nanotubes under ambient conditions. Nat. Nanotechnol. 8, 912-916 (2013).

8. Berman, D., Deshmukh, S. A., Sankaranarayanan, S. K. R. S., Erdemir, A. \& Sumant, A. V. Macroscale superlubricity enabled by graphene nanoscroll formation. Science 348, 1118-1122 (2015).

9. Androulidakis, C., Koukaras, E. N., Paterakis, G. \& Trakakis, G. Tunable macroscale structural superlubricity in two-layer graphene via strain engineering. Nat. Commun. 11, 1595 (2020)

10. Vazirisereshk, M. R. et al. Origin of nanoscale friction contrast between supported graphene, $\mathrm{MoS}_{2}$, and a graphene/MoS 2 heterostructure. Nano Lett. 19, 5496-5505 (2019).

11. Zhang, Z. et al. Macroscale superlubricity enabled by graphene-coated surfaces. Adv. Sci. 7, 1903239 (2020).

12. Leven, I., Krepel, D., Shemesh, O. \& Hod, O. Robust superlubricity in graphene/hBN heterojunctions. J. Phys. Chem. Lett. 4, 115-120 (2013).

13. Erdemir, A. \& Eryilmaz, O. Achieving superlubricity in DLC films by controlling bulk, surface, and tribochemistry. Friction 2, 140-155 (2014).

14. Dietzel, D., Brndiar, J., Stich, I. \& Schirmeisen, A. Limitations of structural superlubricity: chemical bonds versus contact Size. ACS Nano 11, 7642-7647 (2017).

15. Berman, D., Erdemir, A. \& Sumant, A. V. Approaches for achieving superlubricity in two-dimensional materials. ACS Nano 12, 2122-2137 (2018).

16. Liu, S. W. et al. Robust microscale superlubricity under high contact pressure enabled by graphene-coated microsphere. Nat. Commun. 8, 14029 (2017).

17. Li, J., Li, J. \& Luo, J. Superlubricity of graphite sliding against graphene nanoflake under ultrahigh contact pressure. Adv. Sci. 5, 1800810 (2018).

18. Chen, M., Briscoe, W. H., Armes, S. P. \& Klein, J. Lubrication at physiological pressures by polyzwitterionic brushes. Science 323, 1698-1701 (2009).

19. Chen, M., Kato, K. \& Adachi, K. Friction and wear of self-mated $\mathrm{SiC}$ and $\mathrm{Si}_{3} \mathrm{~N}_{4}$ sliding in water. Wear 250, 246-255 (2001).

20. Matta, C., Joly-Pottuz, L., De Barros Bouchet, M. I. \& Martin, J. M. Superlubricity and tribochemistry of polyhydric alcohols. Phys. Rev. B 78, 085436 (2008).

21. Deng, M., Zhang, C., Li, J., Ma, L. \& Luo, J. Hydrodynamic effect on the superlubricity of phosphoric acid between ceramic and sapphire. Friction 2, 173-181 (2014).

22. Ge, X. et al. Macroscale superlubricity under extreme pressure enabled by the combination of graphene-oxide nanosheets with ionic liquid. Carbon 151, 76-83 (2019).

23. Nakhanivej, P. et al. Revealing molecular-level surface redox sites of controllably oxidized black phosphorus nanosheets. Nat. Mater. 18, 156-162 (2019).

24. Favron, A. et al. Photooxidation and quantum confinement effects in exfoliated black phosphorus. Nat. Mater. 14, 826-832 (2015)

25. Zhang, T. et al. Degradation chemistry and stabilization of exfoliated few-layer black phosphorus in water. J. Am. Chem. Soc. 140, 7561-7567 (2018).

26. $\mathrm{Xu}, \mathrm{Y} ., \mathrm{Yu}, \mathrm{J}$., Dong, Y., You, T. \& Hu, X. Boundary lubricating properties of black phosphorus nanosheets in polyalphaolefin oil. J. Tribol. T Asme. 141, 072101 (2019).

27. Wang, W., Xie, G. \& Luo, J. Black phosphorus as a new lubricant. Friction 6, 116-142 (2018).

28. Wang, W., Xie, G. \& Luo, J. Superlubricity of black phosphorus as lubricant additive. ACS Appl. Mater. Inter. 10, 43203-44310 (2018).

29. Wu, S. et al. Black phosphorus: degradation favors lubrication. Nano Lett. 18 5618-5627 (2018).

30. Wu, S. et al. Super-slippery degraded black phosphorus/silicon dioxide interface. ACS Appl. Mater. Inter. 12, 7717-7726 (2020)

31. Wood, J. D. et al. Effective passivation of exfoliated black phosphorus transistors against ambient degradation. Nano Lett. 14, 6964-6970 (2014).

32. Luo, W. et al. Surface chemistry of black phosphorus under a controlled oxidative environment. Nanotechnology 27, 434002 (2016)

33. Kuntz, K. L. et al. Control of surface and edge oxidation on phosphorene. ACS Appl. Mater. Inter. 9, 9126-9135 (2017).

34. Ge, S., Zhang, L., Wang, P. \& Fang, Y. Intense, stable and excitation wavelengthindependent photoluminescence emission in the blue-violet region from phosphorene quantum dots. Sci. Rep. 6, 27307 (2016).
35. Long, L. et al. Highly fluorescent and stable black phosphorus quantum dots in water. Small 14, 1803132 (2018).

36. Guo, T. et al. Black phosphorus quantum dots with renal clearance property for efficient photodynamic therapy. Small 14, 1702815 (2018)

37. Tupikina, E. Y., Bodensteiner, M., Tolstoy, P. M., Denisov, G. S. \& Shenderovich, I. G. $\mathrm{P}=\mathrm{O}$ moiety as an ambidextrous hydrogen bond acceptor. J. Phys. Chem. C. 122, 1711-1720 (2018).

38. Ge, X., Li, J., Luo, R., Zhang, C. \& Luo, J. Macroscale superlubricity enabled by the synergy effect of graphene-oxide nanoflakes and ethanediol. ACS Appl. Mater. Inter. 10, 40863-40870 (2018).

39. Long, Y., De Barros Bouchet, M. I., Lubrecht, T., Onodera, T. \& Martin, J. M Superlubricity of glycerol by self-sustained chemical polishing. Sci. Rep. 9, 6286 (2019).

40. Zeng, Q. F., Yu, F. \& Dong, G. N. Superlubricity behaviors of $\mathrm{Si}_{3} \mathrm{~N}_{4} / \mathrm{DLC}$ films under PAO oil with nano boron nitride additive lubrication. Surf. Interface Anal. 45, 1283-1290 (2013).

41. Kwon, $\mathrm{H}$. et al. Untrathin and flat layer black phosphorus fabricated by reactive oxygen and water rinse. ACS Nano 10, 8723-8731 (2016).

42. Wang, Y. et al. Degradation of black phosphorus: a real-time ${ }^{31} \mathrm{P}$ NMR study. $2 D$ Mater. 3, 035025 (2016)

43. Huang, Y. et al. Interaction of black phosphorus with oxygen and water. Chem. Mater. 28, 8330-8339 (2016).

44. Bai, L., Liu, B., Srikanth, N., Tian, Y. \& Zhou, K. Nano-friction behavior of phos phorene. Nanotechnology 28, 355704 (2017).

45. Cui, Z. et al. Atomic-scale friction of black phosphorus: effect of thickness and anisotropic behavior. Adv. Mater. Int. 4, 1700998 (2017).

46. Losi, G., Restuccia, P. \& Righi, M. C. Superlubricity in phosphorene identified by means of ab initio calculations. 2D Mater. 7, 025033 (2020).

47. Levita, G. \& Righi, M. C. Effects of water intercalation and tribochemistry on $\mathrm{MoS}_{2}$ lubricity: an $\mathrm{Ab}$ initio molecular dynamics investigation. Chemphyschem 18 1475-1480 (2017).

48. Restuccia, P., Ferrario, M. \& Righi, M. C. Monitoring water and oxygen splitting at graphene edges and folds: insights into the lubricity of graphitic materials. Carbon 156, 93-103 (2020).

49. Plimpton, S. J. Fast parallel algorithms for short-range molecular dynamics. J. Comp. Phys. 117, 1-19 (1995).

50. Rappé, A. K., Casewit, C. J., Colwell, K. S., Goddard, W. A. \& Skiff, W. M. UFF, a full periodic table force field for molecular mechanics and molecular dynamics simulations. J. Am. Chem. Soc. 114, 10024-10035 (1992).

51. van der Spoel, D., van Maaren, P. J. \& Berendsen, H. J. C. A systematic study of water models for molecular simulation: derivation of water models optimized for use with a reaction field. J. Chem. Phys. 108, 10220-10230 (1998).

\section{ACKNOWLEDGEMENTS}

This work was supported by the National Natural Science Foundation of China (Grant No. 51822505, 51811530014, and 51905299), and National Key Research and Development Program of China (Grant No. 2020YFA0711000).

\section{AUTHOR CONTRIBUTIONS}

X.R. and G.X., C.Z., D.G., and J.L. conceived the superlubricity study of BP. X.R. performed the experiments and analyzed the data. X.Y. performed MD simulations. X.R., G.X., and $X . Y$. co-wrote the manuscript. J.L. helped the data analysis. All authors contributed to discussions.

\section{COMPETING INTERESTS}

The authors declare no competing interests.

\section{ADDITIONAL INFORMATION}

Supplementary information The online version contains supplementary material available at https://doi.org/10.1038/s41699-021-00225-0.

Correspondence and requests for materials should be addressed to G.X. or J.L.

Reprints and permission information is available at http://www.nature.com/ reprints

Publisher's note Springer Nature remains neutral with regard to jurisdictional claims in published maps and institutional affiliations. 
Open Access This article is licensed under a Creative Commons Attribution 4.0 International License, which permits use, sharing, adaptation, distribution and reproduction in any medium or format, as long as you give appropriate credit to the original author(s) and the source, provide a link to the Creative Commons license, and indicate if changes were made. The images or other third party material in this article are included in the article's Creative Commons license, unless indicated otherwise in a credit line to the material. If material is not included in the article's Creative Commons license and your intended use is not permitted by statutory regulation or exceeds the permitted use, you will need to obtain permission directly from the copyright holder. To view a copy of this license, visit http://creativecommons. org/licenses/by/4.0/.

(c) The Author(s) 2021 